\title{
A BRIDGING METHOD FOR GLOBAL OPTIMIZATION
}

\author{
Y. LIU' ${ }^{1}$ and K. L. TEO ${ }^{1}$ \\ (Received 9 February 1998; revised 26 February 1998)
}

\begin{abstract}
In this paper a bridging method is introduced for numerical solutions of one-dimensional global optimization problems where a continuously differentiable function is to be minimized over a finite interval which can be given either explicitly or by constraints involving continuously differentiable functions. The concept of a bridged function is introduced. Some properties of the bridged function are given. On this basis, several bridging algorithm are developed for the computation of global optimal solutions. The algorithms are demonstrated by solving several numerical examples.
\end{abstract}

\section{Introduction}

In the optimization literature, most optimization techniques seek to find local rather than global optimal solutions. Local optimal solutions may not be very useful for many practical optimization problems, as their cost values may be very far from the true optimal one. One representative example is the minimization of a concave function. In the past decade or so, global optimization has received increasing attention due to its practical importance. As a result, many interesting methods have been developed such as the filled function method [3], LLBF methods $[1,7,8]$ and the branch and bound method $[2,4]$.

For a minimization problem without convexity structure, a classical optimization technique often fails to find a global optimal solution. The main reason is that, once a local minimum is reached, the method does not know how to pass a hill (for minimization) or cross a valley (for maximization) so as to find a better local optimum. The filled function method tries to go through a hill (for minimization) so that it does not get stuck at a local minimum point. The branch and bound methods and the LLBF methods try to discard subregions of the feasible region which do not contain a global optimal solution.

\footnotetext{
'School of Mathematics and Statistics, Curtin University of Technology, Perth, WA 6001, Australia. (C) Australian Mathematical Society 1999, Serial-fee code 0334-2700/99
} 
In this paper we present a new method, called the bridging method, for global optimization. While the name bridging is used for general optimization problems, it comes from the consideration of maximization problems. The idea of the bridging method for maximization is to build a "bridge" over any valley which separates two local maximum points, enabling a certain (local) maximization method to move from one local maximum to a better one. This idea is adapted in this paper in conjunction with a smoothing technique similar to the one developed in [5] for minimization problems involving only a single decision variable. Extension of this result to multidimensional problems, which is currently under investigation, is highly nontrivial and requires substantial effort.

The organization of the paper is as follows. The methods for constructing the right and left bridged functions are introduced in Section 2, where some useful properties of these bridged functions are also given. In Section 3 a computational algorithm is developed based on the results presented in Section 2. The efficiency of the algorithm is illustrated through solving numerous numerical examples. Some numerical results are presented in Section 4. Concluding remarks are given in Section 5.

\section{Construction of bridged functions}

An important part of the bridging method is the construction of the bridged functions. Suppose we have reached a local minimum using a certain local optimization algorithm. We want to build a bridged function to replace the original cost function so that the algorithm is able to move from the present local minimum point to a better local minimum if the current local minimum is not globally optimal. Such a bridged function should have the smoothness required by the algorithm in use and the current point is no longer a local minimum point of the bridged function.

Let $f(x)$ be a given continuously differentiable function on a given interval $[a, b]$. For any $x_{0} \in[a, b], \varepsilon>0$ and $\delta>0$, let

$$
r\left(x, x_{0}, \delta\right)=f\left(x_{0}\right)-\delta\left(x-x_{0}\right)
$$

and

$$
l\left(x, x_{0}, \delta\right)=f\left(x_{0}\right)+\delta\left(x-x_{0}\right) .
$$

For simplicity, we write $r(x)$ for $r\left(x, x_{0}, \delta\right)$ and $l(x)$ for $l\left(x, x_{0}, \delta\right)$ when there is no 
ambiguity. Now define two functions, $f_{r}\left(x, x_{0}, \varepsilon, \delta\right)$ and $f_{l}\left(x, x_{0}, \varepsilon, \delta\right)$, as follows:

$$
\begin{aligned}
& f_{r}\left(x, x_{0}, \varepsilon, \delta\right)= \begin{cases}r(x), & \text { if } x \leq x_{0} \text { or } f(x) \geq r(x), \\
r(x)-(r(x)-f(x))^{2} / 2 \varepsilon, & \text { if } x>x_{0} \text { and } \\
f(x)+\varepsilon / 2, & r(x)-\varepsilon<f(x)<r(x),\end{cases} \\
& f_{l}\left(x, x_{0}, \varepsilon, \delta\right)= \begin{cases}l(x), & \text { if } x>x_{0} \text { and } f(x) \leq r(x)-\varepsilon, \\
l(x)-(l(x)-f(x))^{2} / 2 \varepsilon, & \text { if } x \geq x_{0} \text { or } f(x) \geq l(x), \\
f(x)+\varepsilon / 2, & l(x)-\varepsilon<f(x)<l(x),\end{cases}
\end{aligned}
$$

DEFINITION 2.1. The function $r\left(x, x_{0}, \delta\right)$ (respectively, $l\left(x, x_{0}, \delta\right)$ ) is called a right (respectively, left) bridging function. The function $f_{r}\left(x, x_{0}, \varepsilon, \delta\right)$ (respectively, $f_{l}\left(x, x_{0}, \varepsilon, \delta\right)$ ) is called a right (respectively, left) bridged function at $x_{0}$. Both $f_{r}\left(x, x_{0}, \varepsilon, \delta\right)$ and $f_{l}\left(x, x_{0}, \varepsilon, \delta\right)$ are called bridged functions at $x_{0}$. The point $x_{0}$ is called the bridging point, $\varepsilon$ is called the smoothing constant and $\delta$ is called the bridging slope of the bridged functions $f_{r}\left(x, x_{0}, \varepsilon, \delta\right)$ and $f_{l}\left(x, x_{0}, \varepsilon, \delta\right)$.

DEFINITION 2.2. For given $x_{0}, \varepsilon$ and $\delta$, a subinterval of $[a, b]$ on which $f_{r}\left(x, x_{0}, \varepsilon\right.$, $\delta)=r\left(x, x_{0}, \delta\right)$ is said to be under bridge. Let $\left[r_{1}, r_{2}\right] \subset\left[x_{0}, b\right]$ be an interval under bridge. If no other interval contained in $\left[x_{0}, b\right]$ and strictly containing $\left[r_{1}, r_{2}\right]$ is still under bridge, we call $\left[r_{1}, r_{2}\right]$ a right bridging interval of $f_{r}\left(x, x_{0}, \varepsilon, \delta\right)$. Let $\left[r_{1}, r_{2}\right]$ be a right bridging interval of $f_{r}\left(x, x_{0}, \varepsilon, \delta\right)$. The restriction of $f_{r}\left(x, x_{0}, \varepsilon, \delta\right)$ on $\left[r_{1}, r_{2}\right]$ is called a right bridge of $f_{r}\left(x, x_{0}, \varepsilon, \delta\right)$.

We can introduce the concepts of a left bridging interval and a left bridge for $f_{l}\left(x, x_{0}, \varepsilon, \delta\right)$ similarly. We are specifically interested in the right bridge of $f_{r}\left(x, x_{0}\right.$, $\varepsilon, \delta)$ and the left bridge of $f_{l}\left(x, x_{0}, \varepsilon, \delta\right)$ starting from $x_{0}$. We see that a right bridge of $f_{r}\left(x, x_{0}, \varepsilon, \delta\right)$ is a segment of this function on $\left[x_{0}, b\right]$ where it equals $r\left(x, x_{0}, \delta\right)$. Similarly, a left bridge of $f_{l}\left(x, x_{0}, \varepsilon, \delta\right)$ is a segment of this function on $\left[a, x_{0}\right]$ where it equals $l\left(x, x_{0}, \delta\right)$. In the following, we give some properties of the bridged functions.

LEMMA 2.1. For any $x_{0} \in[a, b], \varepsilon>0$ and $\delta>0$, we have

$$
f_{r}\left(x, x_{0}, \varepsilon, \delta\right) \leq f(x)+\varepsilon / 2, \quad x \in\left[x_{0}, b\right]
$$

and

$$
f_{l}\left(x, x_{0}, \varepsilon, \delta\right) \leq f(x)+\varepsilon / 2, \quad x \in\left[a, x_{0}\right]
$$


PROOF. The result follows directly from the definition of the right and left bridged functions.

LEMMA 2.2. Let $f(x)$ be continuously differentiable on $[a, b]$. Then for any $a \leq$ $x_{0} \leq b, \varepsilon>0$ and $\delta>0$, the bridged functions $f_{r}\left(x, x_{0}, \varepsilon, \delta\right)$ and $f_{l}\left(x, x_{0}, \varepsilon, \delta\right)$ are continuous and continuously differentiable on $[a, b]$. Furthermore,

$$
\frac{\partial}{\partial x} f_{r}\left(x, x_{0}, \varepsilon, \delta\right)= \begin{cases}-\delta, & \text { if } x \leq x_{0} \text { or } f(x) \geq r(x), \\ -\delta+(r(x)-f(x)) \frac{\delta+f^{\prime}(x)}{\varepsilon}, & \text { if } x>x_{0} \text { and } \\ & r(x)-\varepsilon<f(x)<r(x), \\ f^{\prime}(x), & \text { if } x>x_{0} \text { and } \\ & f(x) \leq r(x)-\varepsilon,\end{cases}
$$

and

$$
\frac{\partial}{\partial x} f_{l}\left(x, x_{0}, \varepsilon, \delta\right)= \begin{cases}\delta, & \text { if } x \geq x_{0} \text { or } f(x) \geq r(x), \\ \delta+(r(x)-f(x)) \frac{f^{\prime}(x)-\delta}{\varepsilon}, & \text { if } x<x_{0} \text { and } \\ & r(x)-\varepsilon<f(x)<r(x), \\ f^{\prime}(x), & \text { if } x<x_{0} \text { and } \\ & f(x) \leq r(x)-\varepsilon,\end{cases}
$$

where $r(x)$ (respectively, $l(x)$ ) is the right (respectively, left) bridging function at $x_{0}$ with smoothing constant $\varepsilon$ and slope constant $\delta$.

PROOF. The continuity of the right bridged and left bridged functions is clear from their definitions.

For the continuous differentiability, we only give proof for the right bridged function. The proof for the left bridged function can be obtained similarly.

Let $x \in[a, b]$. Consider four possible cases.

1. If $x<x_{0}$ or $f(x)>r(x)$, then $f_{r}\left(x, x_{0}, \varepsilon, \delta\right)=r(x)$ in a neighborhood of $x$. Thus

$$
\frac{\partial}{\partial x} f_{r}\left(x, x_{0}, \varepsilon, \delta\right)=-\delta
$$

and (2.5) holds true in this case.

2. We can similarly show that (2.5) is true if $x>x_{0}$ and $r(x)-\varepsilon<f(x)<r(x)$, or if $x>x_{0}$ and $f(x)<r(x)-\varepsilon$.

3. If $f(x)=r(x)$, by the continuity of $f_{r}\left(x, x_{0}, \varepsilon, \delta\right)$, we see that, for any increment $\Delta x \neq 0$ such that $x+\Delta x \in[a, b]$, when $\Delta x$ is sufficiently small, we have 
$r(x+\Delta x)-\varepsilon<f(x+\Delta x)$. Thus, if $\Delta x$ is small enough, we have

$$
\begin{aligned}
& \frac{f_{r}\left(x+\Delta x, x_{0}, \varepsilon, \delta\right)-f_{r}\left(x, x_{0}, \varepsilon, \delta\right)}{\Delta x} \\
& = \begin{cases}-\delta, & \text { if } x+\Delta x \leq x_{0} \text { or } f(x+\Delta x) \geq r(x+\Delta x), \\
-\delta-\delta^{2} \Delta x / 2 \varepsilon, & \text { if } x+\Delta x>x_{0} \text { and } \\
& r(x+\Delta x)-\varepsilon<f(x+\Delta x)<r(x+\Delta x)\end{cases} \\
& \rightarrow-\delta(\Delta x \rightarrow 0) \text {. }
\end{aligned}
$$

4. If $f(x)=r(x)-\varepsilon$ and $x>x_{0}$, from the continuity of $f_{r}\left(x, x_{0}, \varepsilon, \delta\right)$, we see that when $\Delta x$ is small enough, we have $x+\Delta x>x_{0}$ and $f(x+\Delta x)<r(x+\Delta x)$. Thus, if $\Delta x$ is small enough, we have

$$
\begin{aligned}
& \frac{f_{r}\left(x+\Delta x, x_{0}, \varepsilon, \delta\right)-f_{r}\left(x, x_{0}, \varepsilon, \delta\right)}{\Delta x} \\
& = \begin{cases}\frac{-\delta \Delta x+\varepsilon / 2-(f(x+\Delta x)-f(x)+\delta \Delta x-\varepsilon)^{2} / 2 \varepsilon}{}, & \Delta x(x+\Delta x)-\varepsilon<f(x+\Delta x)<r(x+\Delta x), \\
\frac{f(x+\Delta x)-f(x)}{\Delta x}, & \text { if } r(x)+\Delta x) \leq r(x+\Delta x)-\varepsilon,\end{cases} \\
& = \begin{cases}-\delta+\frac{\varepsilon^{2}-(f(x+\Delta x)-f(x)+\delta \Delta x-\varepsilon)^{2}}{2 \varepsilon \Delta x}, & \text { if } r(x+\Delta x)-\varepsilon<f(x+\Delta x)<r(x+\Delta x), \\
\frac{f(x+\Delta x)-f(x)}{\Delta x}, & \text { if } f(x+\Delta x) \leq r(x+\Delta x)-\varepsilon,\end{cases} \\
& = \begin{cases}-\delta-\frac{(f(x+\Delta x)-f(x)+\delta \Delta x)(f(x+\Delta x)-f(x)-\delta \Delta x-2 \varepsilon)}{2 \varepsilon \Delta x}, \\
\frac{f(x+\Delta x)-f(x),}{\Delta x}, & \text { if } r(x+\Delta x)-\varepsilon<f(x+\Delta x)<r(x+\Delta x),\end{cases} \\
& \rightarrow f^{\prime}(x)(\Delta x \rightarrow 0) \text {. }
\end{aligned}
$$

Now we see that $f_{r}\left(x, x_{0}, \varepsilon, \delta\right)$ is differentiable with respect to $x$ and its derivative is given by (2.5). The continuity of the derivative is clear from (2.5). The proof is complete.

LEMMA 2.3. Let $\varepsilon>0$ and $\delta>0$ be given. Then we have the following.

(a) If $a \leq x_{0}<b$, then all of the local minimum points of $f_{r}\left(x, x_{0}, \varepsilon, \delta\right)$ on $[a, b]$ must be contained in $\left(x_{0}, b\right]$.

(b) If $a<x_{0} \leq b$, then all of the local minimum points of $f_{l}\left(x, x_{0}, \varepsilon, \delta\right)$ must be contained in $\left[a, x_{0}\right)$. 
PROOF. From Lemma 2.2, $\frac{\partial}{\partial x} f_{r}\left(x, x_{0}, \varepsilon, \delta\right)=-\delta$ for all $x \in\left[a, x_{0}\right]$. Therefore, the right bridged function $f_{r}\left(x, x_{0}, \varepsilon, \delta\right)$ is strictly decreasing in $\left[a, x_{0}\right]$ and its local minimum points must lie in $\left(x_{0}, b\right]$. This proves (a). The proof for (b) can be obtained similarly.

\section{LEMMA 2.4. The following statements hold.}

(a) If $x_{1} \in\left(x_{0}, b\right]$ is a local minimum point of $f(x)$ and $f\left(x_{1}\right)<f\left(x_{0}\right)$, then for sufficiently small smoothing constant $\varepsilon>0$ and slope constant $\delta>0, x_{1}$ is a local minimum point of $f_{r}\left(x, x_{0}, \varepsilon, \delta\right)$.

(b) If $x_{1}$ is a local minimum point of $f_{r}\left(x, x_{0}, \varepsilon, \delta\right)$ and

$$
f_{r}\left(x_{1}, x_{0}, \varepsilon, \delta\right) \leq r\left(x_{1}, x_{0}, \delta\right)-\varepsilon / 2,
$$

then $x_{1}$ is a local minimum point of $f(x)$ satisfying $f\left(x_{1}\right)<f\left(x_{0}\right)$.

PROOF. (a) Let $x_{1} \in\left(x_{0}, b\right]$ be a local minimum point of $f(x)$ and $f\left(x_{1}\right)<f\left(x_{0}\right)$. Then for any $\varepsilon$ and $\delta$ such that

$$
0<\varepsilon<\left(f\left(x_{0}\right)-f\left(x_{1}\right)\right) / 2 \text { and } 0<\delta<\left(f\left(x_{0}\right)-f\left(x_{1}\right)\right) / 2\left(b-x_{0}\right) \text {, }
$$

it is easy to check that $x_{1}$ is a local minimum of $f_{r}\left(x, x_{0}, \varepsilon, \delta\right)$.

(b) The result is clear from the definition of $f_{l}\left(x, x_{0}, \varepsilon, \delta\right)$.

We note that if $x_{1}$ is a local minimum point of $f_{r}\left(x, x_{0}, \varepsilon, \delta\right)$ or $f_{l}\left(x, x_{0}, \varepsilon, \delta\right)$, but

$$
f_{r}\left(x, x_{0}, \varepsilon, \delta\right)>r\left(x, x_{0}, \delta\right)-\varepsilon / 2 \text {, }
$$

then $x_{1}$ is not necessarily a local minimum point of $f(x)$.

DEFINITION 2.3. Let $x_{0} \in[a, b]$ be a given point and $g(x)$ be any function defined on $[a, b]$. A global minimum point of $g(x)$ on $\left[x_{0}, b\right]$ (respectively, $\left[a, x_{0}\right]$ ) is called a right (respectively, left) global minimum point of $g(x)$ with respect to $x_{0}$.

LEMMA 2.5. Let $\varepsilon>0$ and $\delta>0$ be any given numbers.

(a) If $x_{1} \in[a, b)$ is a global minimum point of $f_{r}\left(x, x_{0}, \varepsilon, \delta\right)$ such that

$$
f_{r}\left(x_{1}, x_{0}, \varepsilon, \delta\right) \leq r\left(x_{1}, x_{0}, \delta\right)-\varepsilon / 2,
$$

then $x_{1}$ is a right global minimum point for $f(x)$ with respect to $x_{0}$.

(b) If $x_{1}=b$ is a global minimum of $f_{r}\left(x, x_{0}, \varepsilon, \delta\right)$ and $f\left(x_{1}\right) \leq f_{r}\left(x, x_{0}, \varepsilon, \delta\right)-\varepsilon / 2$, then $x_{1}=b$ is a right global minimum point for $f(x)$ with respect to $x_{0}$. 
PROOF. Let $x_{1} \in[a, b)$ be a global minimum point of $f_{r}\left(x, x_{0}, \varepsilon, \delta\right)$ such that (2.9) holds. In order to prove that $x_{1}$ is a right global minimum point, we need only to show that $x_{1}$ is a global minimum point of $f(x)$ over $\left[x_{0}, b\right]$.

From the definition of $f_{r}\left(x, x_{0}, \varepsilon, \delta\right)$, it is clear from (2.9) that

$$
f(\bar{x})=f_{r}\left(x_{1}, x_{0}, \varepsilon, \delta\right)-\varepsilon / 2 .
$$

If $x_{1}$ is not a global minimum point of $f(x)$ over $\left[x_{0}, b\right]$, then there exists a point $\bar{x} \in\left(x_{0}, b\right]$ such that $f(\bar{x})<f\left(x_{1}\right)$. Now we have

$$
f(\bar{x})<f\left(x_{1}\right)=f_{r}\left(x_{1}, x_{0}, \varepsilon, \delta\right)-\varepsilon / 2 .
$$

Therefore, from Lemma 2.1,

$$
f_{r}\left(\bar{x}, x_{0}, \varepsilon, \delta\right) \leq f(\bar{x})+\varepsilon / 2<f_{r}\left(x_{1}, x_{0}, \varepsilon, \delta\right) .
$$

This contradicts the assumption that $x_{1}$ is a global minimum point of $f_{r}\left(x, x_{0}, \varepsilon, \delta\right)$ on $[a, b]$. Part (a) is proved.

Part (b) is obviously true.

Lemma 2.5 singles out the cases when a global minimum point of $f_{r}$ is a right global minimum point of $f$. If (2.9) is not satisfied in (a), $x_{1}$ is generally not a right global minimum of $f$, since then

$$
r\left(x_{1}, x_{0}, \delta\right)>f\left(x_{1}\right)>r\left(x_{1}, x_{0}, \delta\right)-\varepsilon / 2
$$

may occur and hence, in a neighbourhood of $x_{1}$,

$$
f_{r}\left(x, x_{0}, \varepsilon, \delta\right)=r\left(x, x_{0}, \delta\right)-\left(r\left(x, x_{0}, \delta\right)-f(x)\right)^{2} / 2 \varepsilon,
$$

and $x_{1}$ may not even be a local minimum point of $f_{r}\left(x, x_{0}, \varepsilon, \delta\right)$.

LEMMA 2.6. Let $x_{0} \in[a, b)$ be a point such that

$$
f(x) \geq f\left(x_{0}\right), \quad a \leq x<x_{0} .
$$

Let $\varepsilon>0$ be given. If, for any $\delta>0, x^{1}=b$ is the only minimum point of the right bridged function $f_{r}\left(x, x_{0}, \varepsilon, \delta\right)$ over $[a, b]$ and $f_{r}\left(b, x_{0}, \varepsilon, \delta\right) \leq f(b)$, then $x_{0}$ is a global minimum point of $f(x)$.

PROOF. Suppose $x_{0}$ is not a global minimum point of $f(x)$. Then there exists a global minimum point $x^{*}$ such that $x_{0}<x^{*} \leq b$ and $f\left(x^{*}\right)<f\left(x_{0}\right)$. Let $\delta^{*}=$ 
$\left(f\left(x_{0}\right)-f\left(x^{*}\right)\right) / 2(b-a)$. Then $\delta^{*}>0$ and, hence, by the assumption of the lemma, $f_{r}\left(b, x_{0}, \varepsilon, \delta^{*}\right)<f(b)$. On the other hand,

$$
\begin{aligned}
f_{r}\left(b, x_{0}, \varepsilon, \delta^{*}\right) & =f\left(x_{0}\right)-\delta^{*}\left(b-x_{0}\right) \\
& =f\left(x_{0}\right)-\frac{f\left(x_{0}\right)-f\left(x^{*}\right)}{2(b-a)}\left(b-x_{0}\right) \\
& \geq f\left(x_{0}\right)-\left(f\left(x_{0}\right)-f\left(x^{*}\right)\right) / 2 \\
& =\left(f\left(x_{0}\right)+f\left(x^{*}\right)\right) / 2 \\
& >f\left(x^{*}\right) .
\end{aligned}
$$

Thus $x_{0} \neq b$ and we must have $x_{0}<x^{*}<b$. Since $r\left(x, x_{0}, \delta^{*}\right)$ is strictly decreasing, we see from (2.12) that

$$
f\left(x^{*}\right)<f_{r}\left(b, x_{0}, \varepsilon, \delta^{*}\right)=r\left(b, x_{0}, \delta^{*}\right)<r\left(x^{*}, x_{0}, \delta^{*}\right) .
$$

Hence, by the definition of $f_{r}\left(x, x_{0}, \varepsilon, \delta^{*}\right)$, we have

$$
f_{r}\left(b, x_{0}, \varepsilon, \delta^{*}\right)<f_{r}\left(b, x_{0}, \varepsilon, \delta^{*}\right) .
$$

Furthermore, by assumption, $x_{1}=b$ is the only local minimum point of $f_{r}\left(x, x_{0}, \varepsilon, \delta^{*}\right)$ over $[a, b]$. Hence, $b$ is a global minimum point of $f_{r}\left(x, x_{0}, \varepsilon . \delta^{*}\right)$. This contradicts (2.13). Thus $x_{0}$ is a global minimum point of $f(x)$.

REMARK 2.1. We note that all results given in Lemma 2.4-Lemma 2.6 have their corresponding version for the left bridged function.

\section{Bridging algorithm}

The results presented in the previous section leads to several numerical algorithms, called bridging algorithms, for one-dimensional minimization problems. The aim of this section is to present and discuss these algorithms.

This section is divided into two subsections. Section 3.1 considers minimization problems over a given interval. Section 3.2 considers minimization problems subject to inequality constraints.

3.1. Algorithm for minimization over interval Let $[a, b]$ be a given interval and $f(x)$ be a continuously differentiable function on $[a, b]$. Consider the following problem:

$$
\begin{array}{ll}
\text { Problem }\left(\mathbf{P}^{0}\right): & \text { minimize } f(x) \\
& \text { subject to } x \in[a, b] .
\end{array}
$$


In this section, we shall use $x^{*}$ to denote a global minimum solution of the above problem. For any $x_{0} \in[a, b], \varepsilon>0$ and $\delta>0$, we define two minimization problems associated with Problem $\left(\mathrm{P}^{0}\right)$ :

$$
\begin{array}{cl}
\text { Problem }\left(\mathbf{P}_{r}^{0}\left(x_{0}, \varepsilon, \delta\right)\right): & \operatorname{minimize} f_{r}\left(x, x_{0}, \varepsilon, \delta\right) \\
& \text { subject to } x \in[a, b] . \\
\text { Problem }\left(\mathbf{P}_{l}^{0}\left(x_{0}, \varepsilon, \delta\right)\right): & \text { minimize } f_{l}\left(x, x_{0}, \varepsilon, \delta\right) \\
& \text { subject to } x \in[a, b] .
\end{array}
$$

In the above, the functions $f_{r}$ and $f_{l}$ are the bridged functions of $f$ at $x_{0}$. Algorithm (RB) (the right bridging algorithm):

1. Choose an accuracy number $\delta_{0}>0$, a starting number $\delta_{1} \geq \delta_{0}$ and a dividing number $d>1$ for the slope constant $\delta$. Choose a smoothing constant $\varepsilon_{0}>0$. (See the comment following this algorithm for the choice of these numbers.) Let $x^{*}$ be a variable to store the value of the calculated global minimum solution.

2. Set $x_{0}=a, \delta=\delta_{0}, \varepsilon=\varepsilon_{0}$.

3. If $x_{0}=b$, set $x^{*}=b$ and go to Step 8. Else, go to Step 4 .

4. Find the leftmost local minimum solution, denoted by $\bar{x}$, of Problem $\left(\mathrm{P}_{r}^{0}\left(x_{0}\right.\right.$, $\left.\varepsilon_{1}, \delta\right)$ ).

5. If $r\left(\bar{x}, x_{0}, \delta\right)>f_{r}\left(\bar{x}, x_{0}, \varepsilon, \delta\right)>r\left(\bar{x}, x_{0}, \delta\right)-\varepsilon / 2$, set $\varepsilon=r\left(\bar{x}, x_{0}, \delta\right)-$ $f_{r}\left(\bar{x}, x_{0}, \varepsilon, \delta\right), \delta=\delta_{0}$ and go to Step 4. Else, go to Step 6 .

6. If $\bar{x}<b$, set $x_{0}=\bar{x}, \varepsilon=\varepsilon_{0}, \delta=\delta_{1}$ and go to Step 4. Else if $f(b) \leq$ $f_{r}\left(b, x_{0}, \varepsilon, \delta\right)$, set $x^{*}=b$ and go to Step 8. Else, set $\delta=\delta / d$ and go to Step 7 .

7. If $\delta<\delta_{0}$, set $x^{*}=b$ and goto Step 8. Else, set $\varepsilon=\varepsilon_{1}$ and go to Step 4 .

8. Stop.

A few comments are given below to clarify several points in the above algorithm.

First, we note that it is required in Step 4 to find the leftmost local minimum point of the right bridged function over $[a, b]$. A few available local minimization methods such as the Dichotomy method (with directives) [6] can be used for this purpose. We also note that in solving Problem $\left(\mathrm{P}_{r}^{0}\left(x_{0}, \varepsilon, \delta\right)\right), x_{0}$ is strongly suggested to be taken as the starting point.

Secondly, in the above algorithm, the user is required to choose the constants $\delta_{0}, \delta_{1}, \varepsilon_{0}$ and $d$. Here are some suggestions on the choice of these numbers. The numbers $\delta_{0}, \delta_{1}$ and $d$ are closely related. The accuracy number $\delta_{0}$ should be chosen first. It should be big enough so that when $\delta \geq \delta_{0}$, the computer does not treat $-\delta$ as zero. $\left(-\delta\right.$ is the gradient of $f_{r}\left(x, x_{0}, \varepsilon, \delta\right)$ on its bridge. If $-\delta$ is recognized as negative by the computer, a local minimization algorithm can pass the bridge from its left end to its right end to locate a better local minimum.) At the same time, $\delta_{0}$ should be small enough so that any local minimum of $f(x)$ that is smaller than $f\left(x_{0}\right)$ is still 
a local minimum of $f_{r}\left(x, x_{0}, \varepsilon, \delta\right)$ if $\varepsilon$ is sufficiently small. We see that $\delta_{0}$ is problem dependent. It is not known beforehand in general. However, in practice, it can be taken as a positive number such that a local minimum which is larger than the global minimum by no more than $\delta_{0}$ could be considered a global solution. Usually, $\delta_{0}$ is taken to be $10^{-2}$ or $10^{-3}$. The choice of $\delta_{1}$ is important. If it is chosen too large, even a global minimum point $x^{*}$ of $f(x)$ may no longer be a local minimum point of $f_{r}\left(x, x_{0}, \varepsilon, \delta\right)$, since then we may have $f\left(x^{*}\right)>r\left(x^{*}, x_{0}, \delta\right)$; hence $f_{r}\left(x, x_{0}, \varepsilon, \delta\right)=r\left(x, x_{0}, \delta\right)$ near $x^{*}$ and $x^{*}$ is not a local minimum of $f_{r}\left(x, x_{0}, \varepsilon, \delta\right)$. If it is too small, unnecessary local minimum points of $f$ could be found, increasing the computing effort. Usually, $\delta_{1}$ is taken to be 0.1 or 1 . For given $\varepsilon$ and $\delta$ at some stage, if $f_{r}\left(x, x_{0}, \varepsilon, \delta\right)$ has no local minimum point other than $x=b$ and $f(b)>f_{r}(b)$, then the number $d$ is used to decrease $\delta$ to $\delta / d$. If, in this way, $\delta$ is reduced to a value smaller than $\delta_{0}$, then $x_{0}$ can be considered a global minimum point. Usually $d$ is chosen so that $\delta_{1} / d^{k}=\delta_{0}$ with $k=1$ or 2 . A larger $k$ will possibly increase the computing effort. The value of the smoothing constant $\varepsilon_{0}$ is usually chosen between $10^{-2}$ and 1 . If it is too small, the smoothness of $f_{r}\left(x, x_{0}, \varepsilon, \delta\right)$ may become numerically ill-conditioned. On the other hand, if it is too big, the leftmost local minimum points of $f_{r}\left(x, x_{0}, \varepsilon, \delta\right)$ in the interval $\left(x_{0}, b\right)$ may not be minimum points of $f(x)$. This will cause additional computing effort (see Step 5 of the algorithm).

Finally, we note that for any $x_{1} \in[a, b]$, Algorithm (RB) can be used to find the right global minimum of $f(x)$ with respect to $x_{1}$ if we set the value of $x_{0}$ to $x_{1}$ in Step 4.

Algorithm (RB) above uses the right bridged function and starts from the left end point of the feasible interval. Similarly, we can use the left bridged function to devise a left bridging algorithm. We have the following.

Algorithm (LB) (the left bridging algorithm):

1. Choose an accuracy number $\delta_{0}>0$ and a starting number $\delta_{1} \geq \delta_{0}$ for the slope constant $\delta$. Choose a smoothing constant $\varepsilon_{0}>0$ and a constant $d>1$. (These numbers are chosen the same way as in Algorithm (RB).) Let $x^{*}$ be a variable to store the value of the calculated global minimum solution.

2. Set $x_{0}=b, \varepsilon=\varepsilon_{0}, \delta=\delta_{0}$.

3. If $x_{0}=a$, set $x^{*}=a$ and go to Step 8. Else, go to Step 4 .

4. Find the rightmost local minimum solution, denoted by $\bar{x}$, of Problem $\left(\mathrm{P}_{l}^{0}\left(x_{0}\right.\right.$, $\left.\left.\varepsilon_{1}, \delta\right)\right)$ and go to Step 5 .

5. If $l\left(\bar{x}, x_{0}, \delta\right)>f_{l}\left(\bar{x}, x_{0}, \varepsilon, \delta\right)>l\left(\bar{x}, x_{0}, \delta\right)-\varepsilon / 2$, set $\varepsilon=l\left(\bar{x}, x_{0}, \delta\right)-$ $f_{l}\left(\bar{x}, x_{0}, \varepsilon, \delta\right), \delta=\delta_{0}$, go to Step 4. Else, go to Step 6 .

6. If $\bar{x}<a$, set $x_{0}=\bar{x}, \varepsilon=\varepsilon_{0}, \delta=\delta_{1}$ and go to Step 4. Else, if $f(a) \leq$ $f_{l}\left(a, x_{0}, \varepsilon, \delta\right)$, set $x^{*}=a$ and go to Step 8 . Else, set $\delta=\delta / d$ and go to Step 7 .

7. If $\delta<\delta_{0}$, set $x^{*}=a$ and go to Step 8. Else, go to Step 4 .

8. Stop. 
Comments similar to the ones given for Algorithm (RB) are available for Algorithm (LB). We omit the details to avoid redundancy.

We note that both algorithms require the computation of the local minimum point of the bridged functions that is closest to the bridging point. The efficiency of both algorithms depends on the method used to calculate this local minimum point. When the function $f(x)$ has more than one global minimum point over $[a, b]$, Algorithm (RB) can be used to determine the leftmost global minimum point and Algorithm (LB) to determine the rightmost one. If a third global minimum point exists, it can be determined by using both algorithms starting from a point between the leftmost and the rightmost global minimum points. In this way, all the global minimum points (if there are finitely many) can be found.

3.2. Problems with inequality constraints Let $f(x)$ and $g_{i}(x), i=1,2, \ldots, m$ be $m+1$ continuously differentiable functions on $R^{1}$. Consider the following problem.

$$
\begin{array}{ll}
\text { Problem (P): } & \text { Minimize } f(x) \\
& \text { subject to } g_{i}(x) \leq 0, i=1,2, \ldots, m .
\end{array}
$$

We assume that the feasible region of Problem $(\mathrm{P})$ is a finite interval. For any feasible point $x_{0}$ of Problem (P) and numbers $\varepsilon>0$ and $\delta>0$, we define two problems associated with Problem (P):

$$
\begin{array}{ll}
\text { Problem }\left(\mathbf{P}_{r}\left(x_{0}, \varepsilon, \delta\right)\right): & \text { Minimize } f_{r}\left(x, x_{0}, \varepsilon, \delta\right) \\
& \text { subject to } g_{i}(x) \leq 0, i=1,2, \ldots, m . \\
\text { Problem }\left(\mathbf{P}_{l}\left(x_{0}, \varepsilon, \delta\right)\right): & \text { Minimize } f_{l}\left(x, x_{0}, \varepsilon, \delta\right) \\
& \text { subject to } g_{i}(x) \leq 0, i=1,2, \ldots, m .
\end{array}
$$

Here $f_{r}$ and $f_{l}$ are, respectively, the right and left bridged functions.

The concept of right and left global minimum point introduced in Section 2 can be extended to the current situation. Let $\mathscr{F}$ be the feasible interval of Problem (P). For a given feasible point $x_{0} \in \mathscr{F}$, a point $x_{r} \in \mathscr{F} \cap\left[x_{0}, \infty\right)$ is said to be a right global minimum solution of Problem (P) with respect to $x^{0}$ if $x_{r}$ is a global minimum point of $f(x)$ over $\mathscr{F} \cap\left[x_{0}, \infty\right)$. Similarly, a point $x_{l} \in \mathscr{F} \cap\left(-\infty, x_{0}\right]$ is said to be a left global minimum solution of Problem (P) with respect to $x^{0}$ if $x_{l}$ is a global minimum point of $f(x)$ over $\mathscr{F} \cap\left(-\infty, x_{0}\right]$. Let $x^{0}$ be any feasible point of Problem (P). The idea of the bridging method for Problem (P) is to find a right global minimum solution $x_{r}$ of Problem (P) with respect to $x^{0}$, then find a left global minimum solution $x^{*}$ of Problem (P) with respect to $x_{r}$. The point $x^{*}$ is a global minimum solution of Problem (P).

Thus, in order to solve Problem (P), our main task is to find right and left global minimum solutions given an initial feasible point $x^{0}$. The following procedure can be 
used to calculate the right global minimum solution. It is similar to Algorithms (RB), but differs in that the feasible interval is not explicitly known for the present situation.

The following is a procedure for calculating the right global minimum solution.

1. Choose an accuracy number $\delta_{0}>0$ and a starting number $\delta_{1}>\delta_{0}$ and a dividing constant $d$ for the slope constant $\delta$. Choose a smoothing constant $\varepsilon_{0}>0$ and a constant $d>1$. (These numbers should be chosen in the same way as for Algorithm (RB).) Let $x^{0}$ be a known feasible point and $x_{r}$ be a variable to store the value of the calculated right global minimum solution.

2. Set $x_{0}=x^{0}, \varepsilon=\varepsilon_{0}, \delta=\delta_{1}$.

3. Find the leftmost local minimum solution, denoted by $\bar{x}$, of Problem $\left(\mathrm{P}_{r}\left(x_{0}\right.\right.$, $\varepsilon, \delta)$ ).

4. If $f(\bar{x})=f_{r}\left(\bar{x}, x_{0}, \varepsilon, \delta\right)$, go to Step 7. Else, go to Step 5 .

5. If $f(\bar{x})>f_{r}\left(\bar{x}, x_{0}, \varepsilon, \delta\right)$, set $\delta=\delta / d, \varepsilon=\varepsilon_{0}$ and go to Step 6. Else if $f(\bar{x})>r\left(\bar{x}, x_{0}, \delta\right)-\varepsilon$, set $\varepsilon=r(\bar{x})-f_{r}\left(\bar{x}, x_{0}, \varepsilon, \delta\right), \delta=\delta_{1}$ and go to Step 3 . Else, set $x_{0}=\bar{x}, \varepsilon=\varepsilon_{0}, \delta=\delta_{1}$ and go to Step 3 .

6. If $\delta<\delta_{0}$, set $x^{*}=\bar{x}$ and go to Step 7. Else, set $\varepsilon=\varepsilon_{0}$ and go to Step 3 .

7. Stop.

It is clear that a similar procedure for calculating the left global minimum solution can be obtained by symmetry.

Algorithm (B) (the bridging algorithm):

1. Choose an initial feasible point $x^{0}$. Let $x^{*}$ be a variable to store the value of the computed global minimum point. Set $x_{0}=x^{0}$.

3. Find a right global minimum solution (with respect to $x^{0}$ ), denoted by $x_{r}$, of Problem (P).

4. Find a left global minimum solution (with respect to $x_{r}$ ), denoted by $x_{l}$, of Problem (P). Set $x^{*}=x_{l}$.

5. Stop.

We note that when looking for the left global minimum point of Problem (P) in Step 4 , the point $x^{0}$ can be taken as the starting point although it may be true that $x^{0}<x_{r}$. The reason is that the only possible feasible left global minimum solution of Problem (P) (with respect to $\left.x_{1}\right)$ in $\left(x^{0}, \infty\right)$ is $x_{r}$ and any feasible local minimum point of Problem $\left(P_{l}\left(x_{1}, \varepsilon, \delta\right)\right)$ must be less than or equal to $x^{0}$.

\section{Numerical examples}

The bridging method has been tested by successfully solving many numerical examples including the ones presented in [7]. In this section we present some of the 


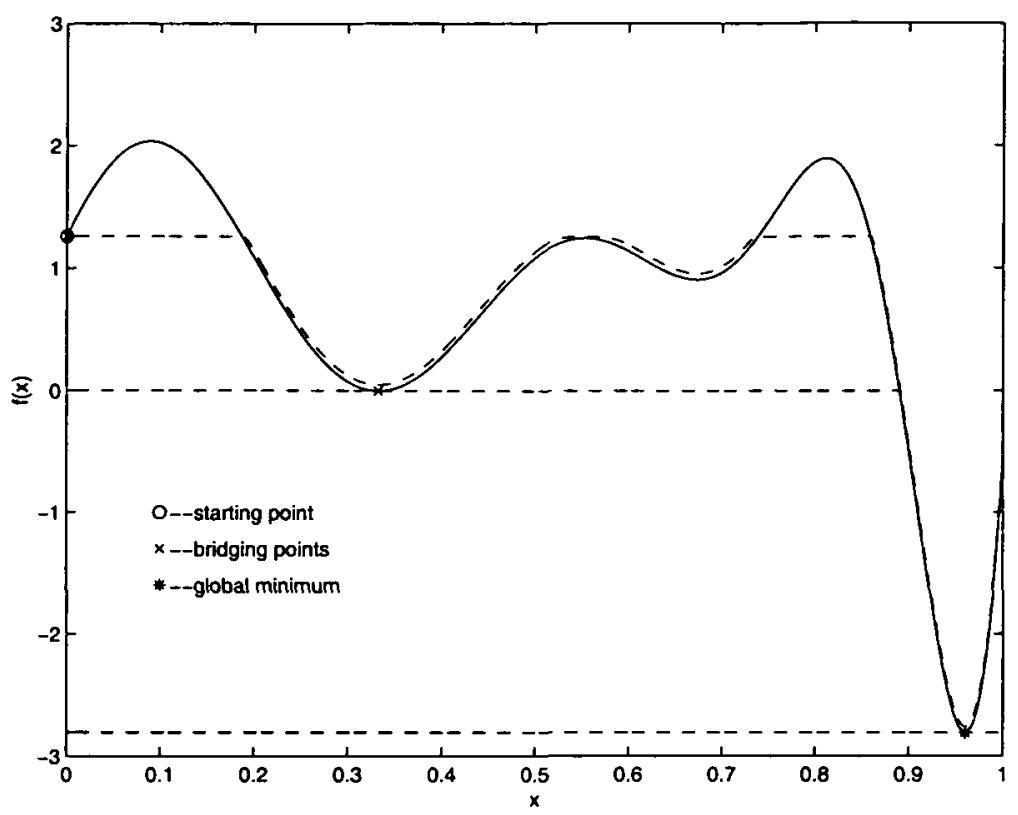

FIGURE 4.1. Minimization procedure for Example 4.1.

test results. For each of the examples, the plot for the whole minimization procedure is given to show how the method works.

EXAMPLE 4.1. $a=0, b=3$ and

$$
\begin{aligned}
f(x)= & (3 x-1) \sin \left(9 x^{2}-3 x\right) \\
& +3(x-1)(3 x-2)^{2}(3 x+0.5)^{3} \cos \left(9 x^{2}-6 x+(\pi+2) / 2\right) .
\end{aligned}
$$

EXAMPLE 4.2. $a=-5, b=-3$ and

$$
f(x)=\frac{1}{4 \pi}\left(\frac{x}{5}-3\right)^{3}\left(\frac{x}{5}+1\right) \cos (x-3) e^{(x / 5+1.2) \sin (\pi x+\pi-5)} .
$$

EXAMPLE 4.3. $a=-10, b=10$ and

$$
f(x)=-e^{0.1 x} \sum_{i=1}^{5} \sin ((i+1) x+i) .
$$

EXAMPLE 4.4. $g_{1}(x)=x^{2}-4 x-21, g_{2}(x)=-e^{x}-x$ and

$f(x)=\frac{5}{(4-x)^{2}+0.5}(\sin (2 x-1)(\cos x+1)+\cos (3 x+2)(\sin x+1))$. 


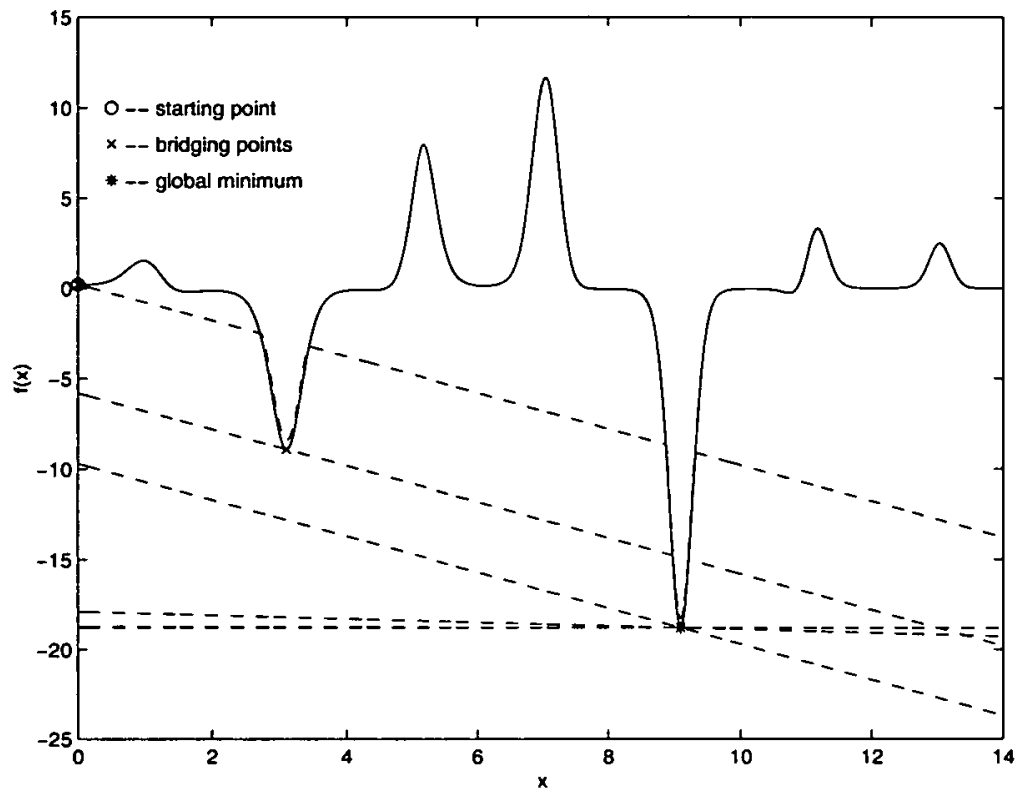

FIGURE 4.2.1. Minimization procedure by right-bridging for Example 4.2.

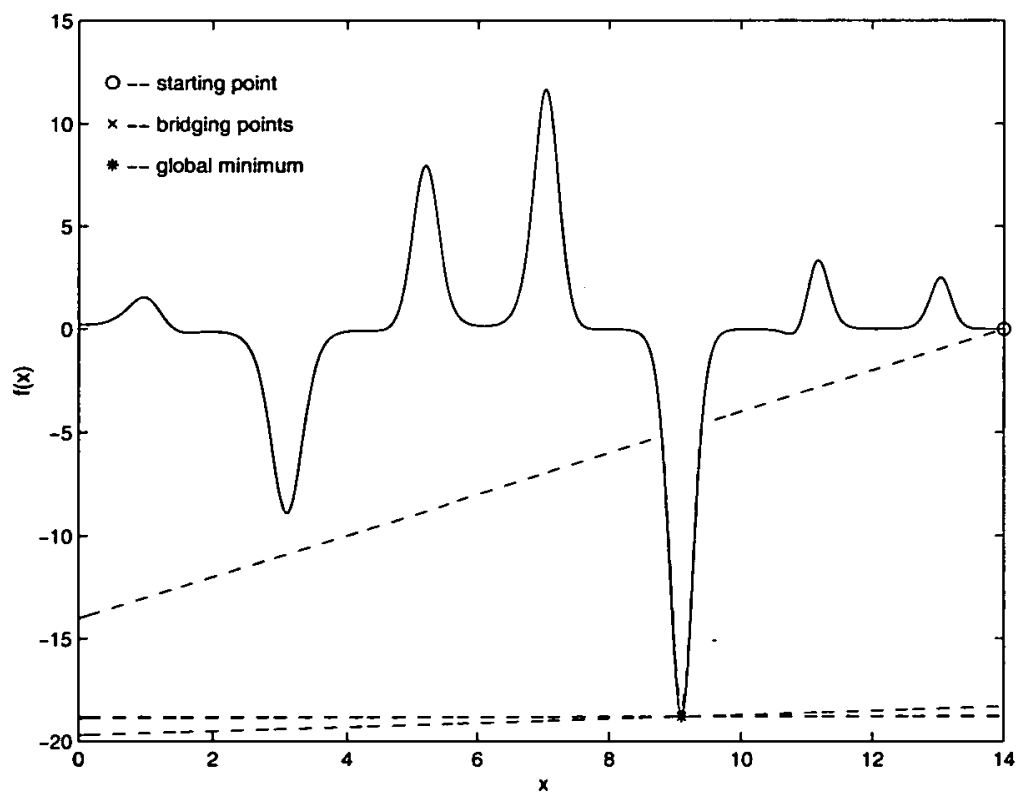

FIGURE 4.2.2. Minimization procedure by left-bridging for Example 4.2. 


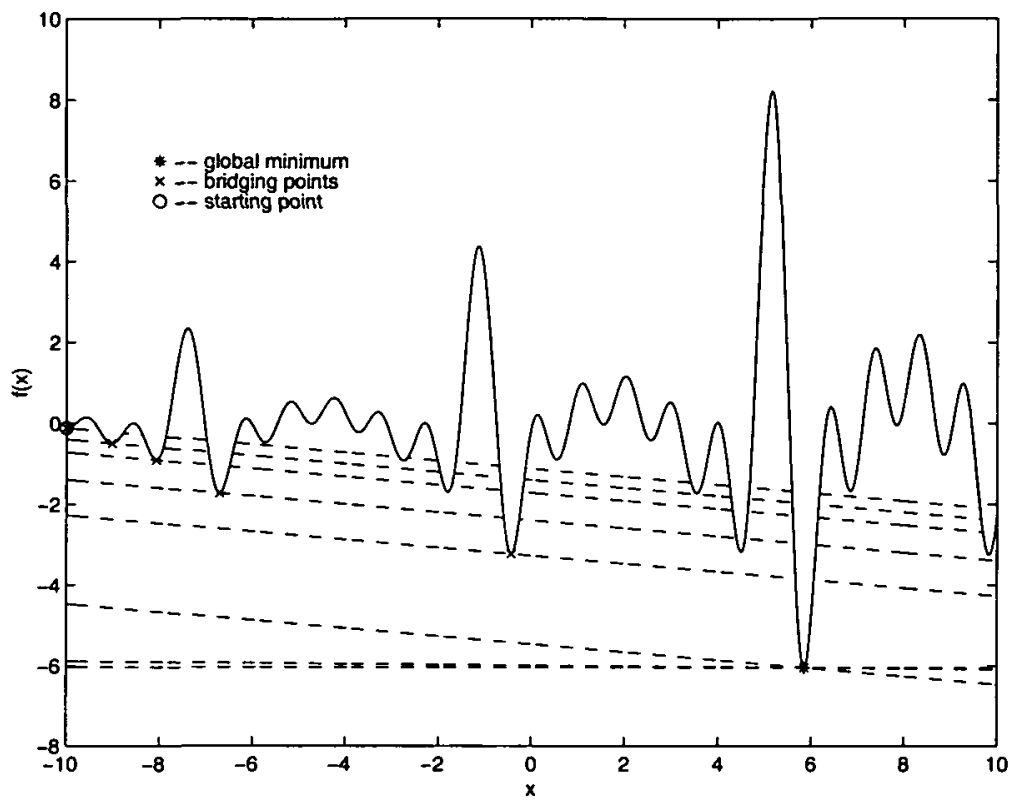

FIGURE 4.3. Minimization procedure for Example 4.3.

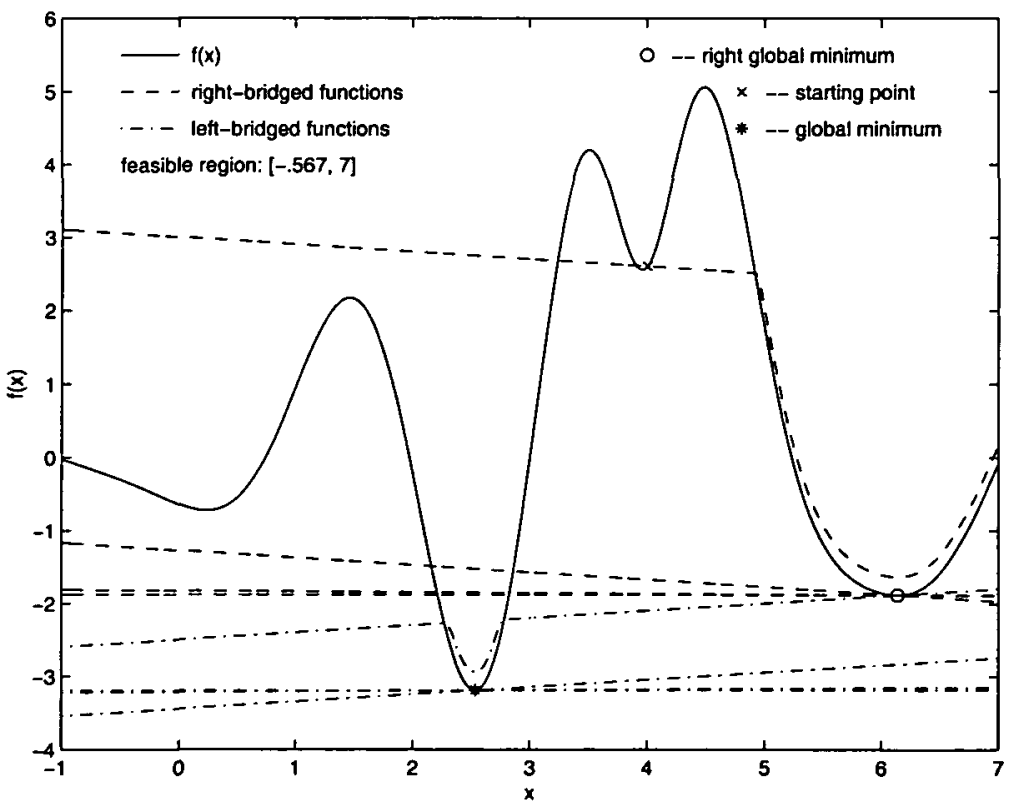

FIGURE 4.4. Minimization procedure for Example 4.4. 
TABLE 4.1. Numerical results for Examples 4.1-4.4.

\begin{tabular}{clrcrccc}
\hline Example & Algorithm & \multicolumn{1}{c}{$\varepsilon_{0}$} & \multicolumn{1}{c}{$\delta_{0}$} & \multicolumn{1}{c}{$\delta_{1}$} & \multicolumn{1}{c}{$d$} & \multicolumn{1}{c}{$x^{*}$} & $f\left(x^{*}\right)$ \\
1 & right bridging & 0.1 & 0.01 & 0.01 & 10 & 0.96046 & -2.80927 \\
2 & right bridging & 1 & 0.001 & 1 & 10 & 9.09475 & -18.79307 \\
2 & left bridging & 1 & 0.001 & 1 & 10 & 9.09475 & -18.79307 \\
3 & right bridging & 0.01 & 0.001 & 0.1 & 10 & 5.85114 & -6.05355 \\
4 & bridging & 0.5 & 0.001 & 0.1 & 10 & 2.53334 & -3.18852 \\
\hline
\end{tabular}

Examples $4.1-4.3$ are solved using the right bridging algorithm. Example 4.2 is also solved by the left bridging algorithm. In finding the leftmost or rightmost local minimum points for each example, we have used the dichotomy method. Example 4.4 is solved using the bridging algorithm in Section 3.2. Although the feasible interval can be easily determined from the given constraints, we didn't use this information in solving the problem.

We note that all the examples presented in [7] can be easily solved by the bridging method. Comparisons on computing time with other global minimization methods are, however, not made since the computing time for the bridging method depends on the method used for minimizing the bridged functions. In general, the total time used to obtain a global solution is approximately the total time used to minimize the bridge functions. The computation results are listed in Table 4.1. The whole computing procedure for all of the four examples are shown in Figures 4.1-4.4. For Example 4.2, the procedures corresponding to the right and left bridging algorithms are shown in Figures 4.2.1 and 4.2.2, respectively. The solid line in each figure represents the original cost function. The broken lines represent the right bridged functions and the dotted broken lines the left bridged functions used in the minimization procedure.

\section{Conclusion}

In this paper, a bridging method for one-dimensional optimization problems is proposed and several bridging algorithms are developed for both simply bounded and analytically constrained problems. Numerical experiment has shown that the bridging method is efficient in locating a global optimal solution. This method has the potential of being generalized or applied to multi-dimensional problems, though some features appearing in the one-dimensional case may not be preserved. Further research in this direction is under consideration. 


\section{Acknowledgement}

This research is partially supported by a research grant from the Australian Research Council.

\section{References}

[1] M. Bromberg and T-S. Chang, "One dimensional optimization using linear lower bounds", in Recent Advances in Global Optimization (eds C. A. Floudas and P. M. Pardalos), (Princeton University Press, 1992) 200-220.

[2] C. A. Floudas and P. M. Pardalos (eds), State of the art in Global Optimization (Kluwer Academic Publishers, Dordrecht, 1996).

[3] R. Ge, "A filled function method for finding a global minimizer of a function of several variables", Math. Programming 46 (1990) 191-204.

[4] R. Horst, P. M. Pardalos and N. V. Thoai, Introduction to Global Optimization (Kluwer Academic Publishers, Dordrecht, 1995).

[5] L. S. Jennings and K. L. Teo, "A computational algorithm for functional inequality constrained optimization problems", Automatica 26 (1990) 371-375.

[6] M. Minoux, Mathematical Programming, Theory and Applications (John Wiley and Sons, Chichester, 1986).

[7] X. Wang and T.-S. Chang, "An improved univariate global optimization algorithm with improved linear lower bounding functions", J. Global Optimization 8 (1996) 393-411.

[8] X. Wang and T-S. Chang, "A multivariate global optimization using linear bounding functions", 1997 , preprint. 